\title{
A comprehensive discussion on colour conversion element design of phosphor converted LEDs
}

\author{
Susanne Schweitzer ${ }^{1}$, Christian Sommer ${ }^{1}$, Paul Hartmann ${ }^{1}$, Paul Fulmek², Johann Nicolics ${ }^{2}$, Peter Pachler ${ }^{3}$, \\ Hans Hoschopf ${ }^{3}$, Franz Schrank ${ }^{3}$, Gregor Langer ${ }^{4}$ and Franz P Wenzl ${ }^{1 *}$
}

\author{
* Correspondence: \\ Franz-Peter.Wenzl@joanneum.at \\ ${ }^{1}$ Institute of Surface Technologies \\ and Photonics, Joanneum Research, \\ Forschungsgesm.b.H., Franz-Pichler \\ Straße 30, A-8160 Weiz, Austria \\ Full list of author information is \\ available at the end of the article
}

\begin{abstract}
For a systematic approach to improve the white light quality of phosphor converted light-emitting diodes (LEDs) for general lighting applications it is imperative to get the individual sources of error for correlated colour temperature (CCT) reproducibility and maintenance under control. In this regard, it is of essential importance to understand how geometrical, optical and thermal properties of the colour conversion elements (CCEs), which typically consist of phosphor particles embedded in a transparent matrix material, affect the constancy of a desired CCT value. In this contribution we use an LED assembly consisting of an LED die mounted on a printed circuit board by chip-on-board technology and a CCE with a globe-top configuration on the top of it as a model system and discuss the impact of the CCE size, the substrate reflectivity as well as the thermal load of the CCEs on CCT maintenance and the respective radiant fluxes. It turns out that optimized solutions for CCT maintenance and high radiant fluxes in regard of one of the relevant influence factors do not need to be optimized solutions in regard of another one.
\end{abstract}

\section{Background}

Although the phenomenon of electroluminescence is known for more than 100 years $[1,2]$, it took till the early 1990s that efficient blue LEDs, which are the core elements for solid state lighting (SSL), have been available [3,4]. From that time on, a great amount of worldwide industrial and academic research activities resulted in a rapid enhancement of both device performance and life time. Nonetheless, as noted, e.g., in [5], estimates are that SSL has only reached the halfway point in terms of lighting efficiency, which leaves a lot of challenges ahead-not just in terms of energy efficiency, but also for colour quality and control, as well as other potential advantages which are largely untapped [5].

For example, it has been concluded that for white LEDs to be commonly accepted for general lighting applications, the colour variation among individual LEDs should become of the order of a 2-step MacAdam ellipse [6]. For several reasons it is hard to meet this demand with today's most common approach for white LEDs, which relies on the excitation of a phosphor by blue or UV emitting LEDs. The phosphor is part of the colour conversion element (CCE), which typically consists of phosphor particles embedded in a silicone matrix. While this concept seems to be rather trivial, recent 
studies have highlighted that the lumen output and the white light quality of phosphor converted LEDs critically depend on an appropriate design of the CCEs and are strongly affected by their shape, composition and arrangement within the LED package [7-24].

As shown recently, besides its direct impact on the optical properties, the CCE design has also a strong impact on the thermal load of the CCEs during device operation. Even in the case that most of the heat is generated in the LED die, in today's LED packages the highest temperatures are often located in the CCE as a consequence of the low thermal conductivity of the silicone matrix [25]. Besides the related risk for long-term materials degradation, this in particularly also has a strong impact on correlated colour temperature $(\mathrm{CCT})$ maintenance. Since phosphors are generally prone to decreasing luminescence intensity with increasing temperature, which means that the overall contribution of the yellow emission decreases, one has to ensure that the temperature increase and variation within the LED package and in particular in the CCE upon device operation is as low as possible. Using one specific Ce doped yttrium aluminum garnet (Ce:YAG) as a reference phosphor, in a recent study it has been concluded that a temperature variation of about $40 \mathrm{~K}$ might be just tolerable to limit variations of the CCT values within a MacAdam ellipse of step 2 [26].

Together with the manufacturing and fabrication processes of the LED packages and their components, which involve a lot of potential failure sources, these are challenging demands which all may influence a desired CCT value and a deviation therefrom. In principle, the shape and the composition of the CCEs can be designed to alleviate the impact of most of the parameters affecting the constancy of a CCT value in each case. However, for a concerted consideration it is often necessary to make compromises in regard of optimized CCE designs, since the optimization of the CCE with respect to one of the influencing factors may negatively affect another one. In a recent publication, we have highlighted this based on a consideration of manufacturing imperfections of colour conversion elements in globe-top configurations [27]. In the following, we extend these studies and show that in dependency of the substrate surface reflectivity and the sizes of globe-tops (which have a hemispherical shape) optimized solutions for CCT maintenance in regard of one of the relevant parameters do not need to be optimized solutions in regard of another one.

\section{Methods}

The details of the optical simulation procedure can be found in our previous publication [15]. Generally, this simulation procedure, which was carried out with the commercial software package ASAP $^{\mathrm{m}}$, relies on the set-up of an appropriate simulation model for a blue emitting LED die and the implementation of a CCE in the direction of the blue light emitted from the die (see Figure 1 for a sketch of the LED assembly). The die's active area $(940 \mu \mathrm{m} \times$ $940 \mu \mathrm{m})$ is located on top of a silicon substrate with lateral dimensions of $990 \mu \mathrm{m} \times$ $990 \mu \mathrm{m}$ and a height of $100 \mu \mathrm{m}$. This specific die design refers to a vertical thin film LED die like the EZ 1000 (Gen I) from Cree [28]. From the data sheet of this chip, also the electrical and optical characteristics (voltage, current, corresponding radiant flux...) for the LED die under operation were deduced. The LED die is mounted on a printed circuit board (PCB) by chip-on-board technology with an adhesive layer having a height of $10 \mu \mathrm{m}$. The PCB consists of an aluminium substrate with a height of $1500 \mu \mathrm{m}$ and a dielectric layer $(80 \mu \mathrm{m})$ as well as a copper layer $(70 \mu \mathrm{m})$ on its top. 


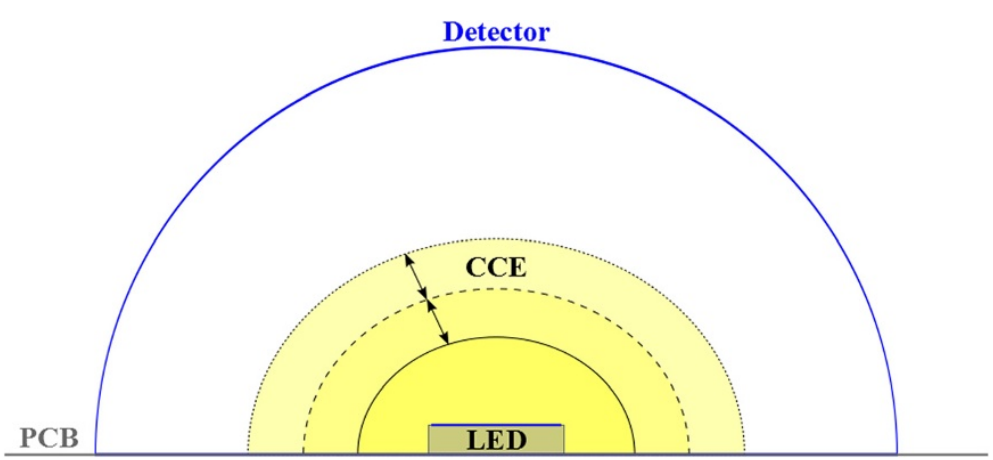

Figure 1 The model of the LED assembly consists of a blue emitting LED die with a CCE in globe-top configuration surrounding the die. The irradiance distributions for both the blue LED light and the yellow converted light are monitored by a detector, which has a hemispherical shape and is centrically placed above the LED package. The die's active area $(940 \mu \mathrm{m} \times 940 \mu \mathrm{m})$ is located on top of a silicon substrate which has lateral dimensions of $990 \mu \mathrm{m} \times 990 \mu \mathrm{m}$ and a height of $100 \mu \mathrm{m}$. The silicon substrate is mounted on a printed circuit board by chip-on-board technology. The CCEs are assumed to have a globe-top configuration with a hemispherical shape and radii which vary from $1 \mathrm{~mm}$ to $2 \mathrm{~mm}$ and $3 \mathrm{~mm}$. The phosphor concentrations for these three different globe-top sizes are adjusted in order to get similar overall CIE $x$ values of about 0.3300 and 0.3800 in each case, under the assumption that the substrate surface has a diffuse reflectivity of $85 \%$.

In the present study, the CCE is assumed to have a globe-top configuration with a hemispherical shape. In order to study the impact of different globe-top sizes on the optical and thermal properties the radii of these hemispheres are varied from $1 \mathrm{~mm}$, to $2 \mathrm{~mm}$ and $3 \mathrm{~mm}$ as schematically shown in Figure 1. The phosphor concentrations for these different globe-top sizes are adjusted in order to get similar CIE $x$ values of about 0.3300 ( 5.115 vol.\% for $1 \mathrm{~mm}, 2.244$ vol.\% for $2 \mathrm{~mm}$ and 1.454 vol.\% for $3 \mathrm{~mm}$ ) and 0.3800 ( 8.373 vol.\% for $1 \mathrm{~mm}, 3.566$ vol.\% for $2 \mathrm{~mm}$ and $2.299 \mathrm{vol} . \%$ for $3 \mathrm{~mm}$ ) in each case, while the substrate surface is assumed to have a diffuse reflectivity of $85 \%$.

Two wavelengths are considered for the optical simulations [15]: one representing the blue LED light $(460 \mathrm{~nm})$, and the other one the converted yellow light $(565 \mathrm{~nm})$. It is assumed that only the blue light is absorbed by the phosphor particles, therefore the extinction coefficient of the phosphor particles is set to zero for $565 \mathrm{~nm}$ and to $1 \times 10^{-3}$ for $\lambda=460 \mathrm{~nm}$. Both the blue LED light and the yellow converted light are scattered throughout the CCE. The simulation of this scattering process is based on the scattering model of Mie and considers the particle size distribution of the phosphor as well as the optical properties of both the matrix material and the phosphor. The refractive indexes of the silicone and the phosphor are kept constant at 1.4 and 1.63, respectively, for both wavelengths. Similarly, the mean diameter of the phosphor particles is kept constant at $7.8 \mu \mathrm{m}$ with a standard deviation of $4.2 \mu \mathrm{m}$. The irradiance distributions both for the blue LED light and the yellow conversion-light are monitored by a detector, which has a hemispherical shape and is centrically placed above the LED package, see Figure 1.

The surface of this detector is divided into $101 \times 101$ pixels (in direction of the $\mathrm{x}$ and $\mathrm{y}$ principle axes). Hence, the projection of this surface of the hemispherical detector onto a flat plane gives a matrix consisting of $101 \times 101$ elements, which e.g., allows one to determine the mean of the CIE $x$ values for two perpendicular directions (line-by-line and column-by-column averaging) of the matrix as a function of the pixel number [15].

Since the present study mainly focuses on the impact of the CCE and the materials it is composed of, it has to be mentioned that potential impacts which are related to the 
LED die itself are not considered. This means that, although the optical properties of the LED die are considered in the simulations, these values are assumed to be constant throughout this study. In reality, the absorption and the reflectivity of the light by the LED die may also change due to some long-term degradation [29].

In order to gain detailed information on the absorption profile of the blue LED light within the CCE, the latter is divided into a number of voxels, each of them having a size of $10 \mu \mathrm{m} \times 10 \mu \mathrm{m} \times 10 \mu \mathrm{m}(10 \mu \mathrm{m}$ in each direction). From the ray-tracing simulations, the amount of the blue radiant flux absorbed in each individual voxel can be determined. With the respective overall radiant flux for a specific current as determined from the data sheet [28], the absolute values of the blue radiant fluxes which are absorbed in each individual voxel can therefore be calculated. These absorption profiles are taken as input data for the subsequent thermal simulations [25,30], for which three-dimensional models of the LED package were set up using the GPL-software packages GetDP/Gmsh [31,32]. In this case, the CCE is modelled as a unit with a specific thermal conductivity (which is determined by the phosphor concentration in the silicone matrix [25]) and heat capacity. Using the symmetry of the model, the calculation effort and time can be reduced by simulating only one eighth of the whole sample. The local power sources of the absorption profile in the CCE, however, break this symmetry. Therefore, an equivalent symmetrical CCE-power-source distribution was determined and verified by comparison with full 3D-simulations. At the symmetry planes of this reduced model space, adiabatic boundary conditions were applied.

Two sources for heat generation within the CCE are considered in this study, on the one hand heat generation due to the Stokes shift for those blue photons which are converted into yellow ones (460 $\mathrm{nm}$ for the absorbed blue photons and $565 \mathrm{~nm}$ for the re-emitted yellow photons) and on the other hand heat generation of those blue photons which are absorbed but which recombine non-radiatively (which is considered for by a quantum efficiency of $70 \%$, which means that 0.7 yellow photons are emitted for each absorbed blue photon) [25]. At the assembly's bottom face (outside the size range presented in the respective figures) Dirichlet boundary conditions with a temperature of $300 \mathrm{~K}$ are assumed. All other boundaries of the model are subject to natural convection in air. The exact value of the coefficient of natural convection $h$ depends on many factors (size of the sample, orientation of the sample in space, ambient temperature, etc.). For the simulations presented in this study, a value of $h=20 \mathrm{~W} /\left(\mathrm{m}^{2} \mathrm{~K}\right)$ [33] and an ambient temperature of $300 \mathrm{~K}$ have been selected.

\section{Results and discussion}

Figure 2 shows the variation of the overall CIE $x$ values in dependency of the reflectivity of the substrate surface (which is assumed to be either of specular or of diffuse nature and which is varied in between $70 \%$ and 100\%) and the globe-top sizes. The phosphor concentrations for the globe-tops with the radii of $1 \mathrm{~mm}, 2 \mathrm{~mm}$ and $3 \mathrm{~mm}$ were adjusted to achieve overall CIE $x$ values of about 0.3300 and 0.3800 (for a diffuse reflectivity of the substrate surface of $85 \%$ ).

In the case of a specular substrate surface reflectivity, the same phosphor concentrations as in case of a diffuse one were used.

An increase of the substrate surface reflectivity to values larger than $85 \%$ goes along with an increase of the overall CIE $x$ values for all three globe-top radii. This can be 

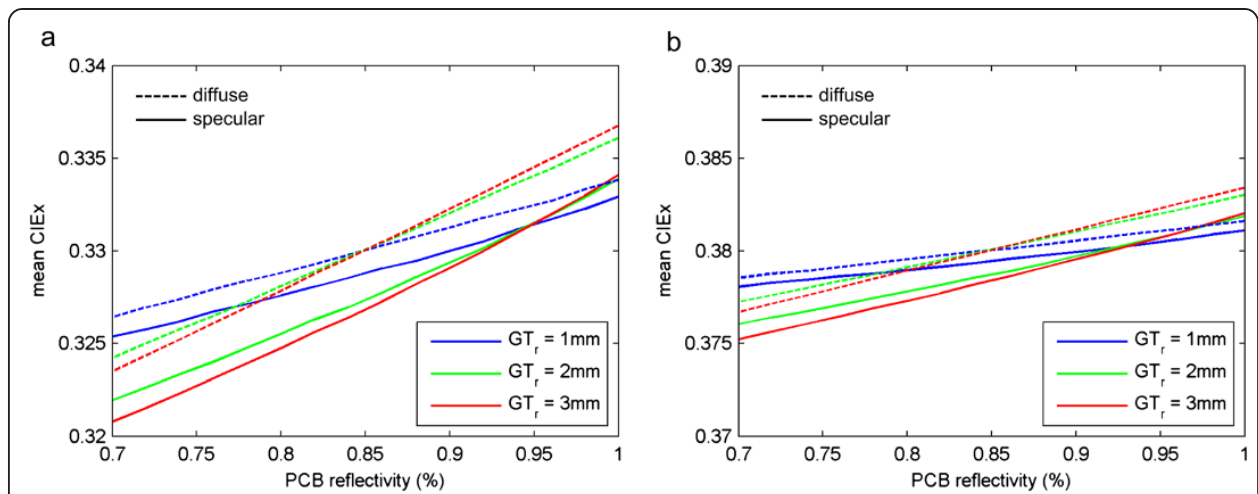

Figure 2 Variation of the overall CIE $x$ values in dependency of the reflectivity of the substrate surface for globe-top configurations with radii of $1 \mathrm{~mm}, 2 \mathrm{~mm}$, and $3 \mathrm{~mm}$. The phosphor concentrations for the different globe-top sizes are adjusted in order to get similar overall CIE $x$ values of about 0.3300 (a) and 0.3800 (b) under the assumption of a diffuse reflectivity of the substrate surface of $85 \%$. The dashed lines show the variation of the CIE $x$ values for a diffuse reflectivity of the substrate surface, the solid lines for a specular reflectivity. $\mathrm{GT}_{\mathrm{r}}$ refers to the globe-top radius.

attributed to the fact that with increasing reflectivity the backscattered light becomes less and less absorbed. Since the yellow light emitted from the phosphor particles is more prone to be backscattered than the blue LED light, the substrate reflectivity in particularly affects the yellowish fraction of the overall emission spectrum and therefore also the overall CIE $x$ values increase with increasing substrate reflectivity. In principle, as suggested in [24], a higher reflectivity of the substrate surface could be therefore applied in order to lower the amount of phosphor which is necessary in order to achieve a desired CCT value. The lowest increase of the CIE $x$ value (and therefore the lowest deviation from the initial value) with increasing substrate reflectivity shows the hemispherical globe-top with the smallest of the three radii since in this case also the coverage of the substrate surface with the globe-top material is the lowest.

This behaviour reverses in case that the reflectivity of the substrate surface is reduced to values lower than $85 \%$. In this case, the overall CIE $x$ values decrease for all the different globe-top radii since a comparably larger amount of the (mainly yellow) backscattered light is absorbed by the substrate surface. Therefore, again, the hemispherical globe-top with the smallest radius shows the lowest decrease of the CIE $x$ values and the lowest deviation from the initial value. In practice, it is most likely that the reflectivity of the substrate surface decreases (and not increases) during the lifetime of the LED assembly because of materials degradation. One can therefore conclude that a smaller globe-top size has some advantages in regard of long-term maintenance of a given colour temperature.

In addition, a comparison of specular and diffuse substrate surface reflectivities shows that for the same value of the reflectivity, a specular reflectivity gives reason for somewhat lower CIE $x$ values than a diffuse one. This behaviour is a bit more pronounced for the larger globe-top radii than for the smaller ones. Since for both types of reflectivity the amount of phosphor is the same (with respect to the individual globe-top radii), the use of diffuse substrate surfaces opens another possibility to reduce the required volume concentration and therefore amount of phosphor to achieve a desired CCT value.

In order to highlight this more in detail, the following approximation can be done, in which hemispheres with the different radii and the respective phosphor concentrations are considered, however the volume of the LED die is neglected. Assuming a density of 
the phosphor of $4.56 \mathrm{~g} / \mathrm{cm}^{3}$ as it is typical for Ce-YAG based systems [34] and of $0.96 \mathrm{~g} / \mathrm{cm}^{3}$, which is an order of range value for PDMS [35], the required amounts of phosphor for the three globe-top radii in case of the diffuse substrate are given by $0.00048 \mathrm{~g}(1 \mathrm{~mm}), 0.00171 \mathrm{~g}(2 \mathrm{~mm})$ and $0.00378 \mathrm{~g}(3 \mathrm{~mm})$ for a CIE $x$ value of 0.33 as well as $0.00079 \mathrm{~g}(1 \mathrm{~mm}), 0.00272 \mathrm{~g}(2 \mathrm{~mm})$ and $0.00592 \mathrm{~g}(3 \mathrm{~mm})$ in the case of a CIE $x$ value of 0.38 . Considering the above mentioned differences for specular (for a CIE $x$ value of 0.33 and a globe-top with a radius of $1 \mathrm{~mm} 0.00049 \mathrm{~g}$, for $2 \mathrm{~mm} 0.00175 \mathrm{~g}$ and $3 \mathrm{~mm} 0.003841 \mathrm{~g}$ would be required) and diffuse substrate reflectivities, this means that in case of a CIE $x$ value of 0.33 and a specular substrate surface reflectivity an about $1 \%$ higher amount of phosphor for the globe-top with the radius of $1 \mathrm{~mm}$ would be required and an about 2.5\% higher amount for the globe-top with the radius of $3 \mathrm{~mm}$.

These differences of diffuse and specular surface reflectivities are also reflected in a comparison of the mean of the CIE $x$ values for two perpendicular directions (line-by-line and column-by-column averaging) of the data matrix as a function of the pixel number as shown in Figures 3 and 4. Figure 3 compares the mean of the CIE $x$ values for a globe-top with a radius of $1 \mathrm{~mm}$ and a substrate surface of either diffuse (left column) or specular (middle column) reflectivity. For a diffuse reflectivity of the substrate surface of $85 \%$, for which the phosphor concentration is adjusted in order to achieve a CIE $x$ value of about 0.3300 , the Figure shows that for normal viewing directions the CIE $x$ values are a bit lower than for larger viewing angles. This is in accordance with a recent study by Sun et al. [14], in which it was concluded that the size of a hemispherical globe-top should be increased in normal direction in order to gain white light-emission with low variation of the colour temperature for different viewing angles. The same trend can be observed in case of a diffuse reflectivity of either $70 \%$ and $100 \%$. For a specular reflectivity and the same conditions, this behaviour is even more pronounced. This means, the CIE $x$ values become comparably lower in particular for normal viewing directions. This is also emphasized in the difference graphs for the mean of the CIE $x$ values as a function of the pixel number for a specular and a diffuse reflectivity as shown in the right column of Figure 3.

The same behaviour can be also observed in case of the globe-top with a radius of $3 \mathrm{~mm}$, for which the difference graphs for diffuse and specular reflectivities of the substrate surface are shown in the left column of Figure 4.

A detailed comparison of these images with the images shown in the right column of Figure 3 highlights that for a globe-top with $3 \mathrm{~mm}$ radius there are in particular more pronounced differences between diffuse and specular reflectivity for larger viewing angles than in the $1 \mathrm{~mm}$ case.

The middle column of Figure 4 shows the difference graphs for the mean of the CIE $x$ values for globe-tops having radii of $1 \mathrm{~mm}$ and $3 \mathrm{~mm}$ for a diffuse reflectivity. While there is no significant difference for a diffuse reflectivity of $85 \%$ (in particular since also the phosphor concentrations were adjusted in order to achieve the same CIE $x$ value of about 0.3300 for the different radii), there is a shift to the bluish with increasing globe-top size for decreasing surface reflectivities and a shift to the yellowish with increasing globe-top size for increasing surface reflectivities. However, for the same type of (diffuse) reflectivity, the globe-top radius has no obvious impact on angular dependent colour variations.

A similar behaviour as in case of phosphor concentrations which give reason for CIE $x$ values of about 0.3300 can also be observed for phosphor concentrations giving reason for CIE $x$ values of about 0.3800 . This is emphasized in the images of the right 


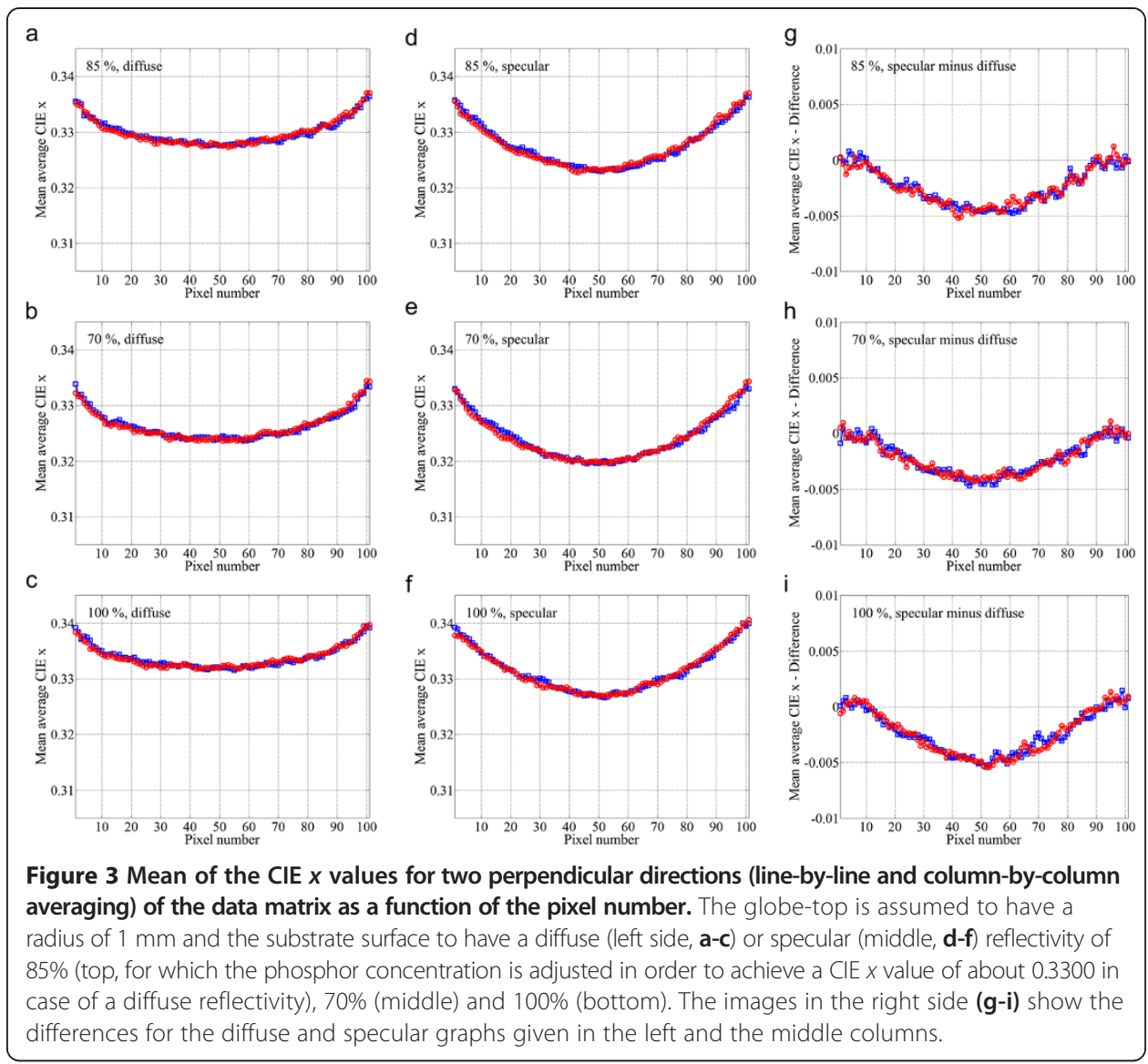

column of Figure 4, which show the difference of the mean of the CIE $x$ values as a function of the pixel number for specular and diffuse reflectivities for globe-top radii of $3 \mathrm{~mm}$.

As a conclusion, these studies show that the colour maintenance is the better the smaller the globe-top radius is and that with a substrate surface having a diffuse reflectivity not only higher CIE $x$ values but also lower angular variations of the CIE $x$ values can be realized.

Figures 5 and 6 finally compare the radiant fluxes (blue radiant flux, yellow radiant flux and total radiant flux) for globe-top sizes of $1 \mathrm{~mm}$ and $3 \mathrm{~mm}$. For this comparison, again a diffuse reflectivity of the substrate surface of $85 \%$ is taken as a reference system. For the same globe-top radii, an increasing substrate surface reflectivity of up to $100 \%$ gives reason for higher radiant fluxes, in particular for the yellow radiant flux, which again is due to the higher contribution of the yellow light on the overall backscattered light. Again, this enhancement is more pronounced for globe-tops with larger radii, since in this case the coverage of the substrate by the globe-top material is also larger.

In addition, a comparison of specular and diffuse reflectivity shows, that the observed lower CIE $x$ values for a specular reflectivity as shown in Figure 2 are mainly caused by a reduction of the yellowish fraction of the light.

Contrarily, in case that the reflectivity of the substrate surface is reduced to $70 \%$, the radiant fluxes decrease. Again, the yellow radiant flux is more affected than the blue one, since a larger portion of the backscattered (mainly yellow) light now becomes absorbed by the substrate surface. 

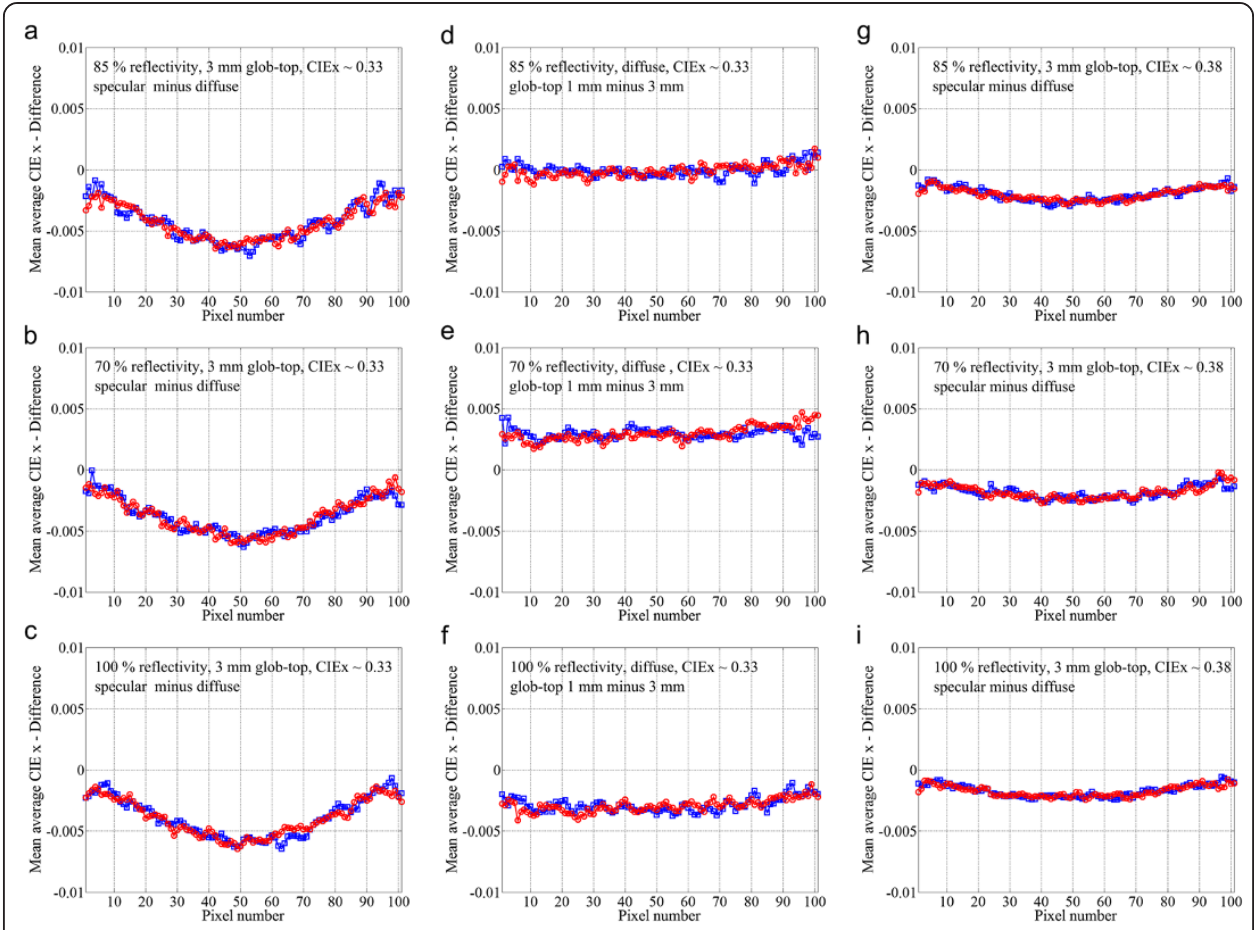

Figure 4 Mean of the CIE $x$ values for two perpendicular directions (line-by-line and column-by-column averaging) of the data matrix as a function of the pixel number. Left side (a-c): Differences of diffuse and specular reflectivity for a globe-top size of $3 \mathrm{~mm}$ (CIE $x$ value of about 0.3300 ). Middle (d-f): Differences for globe-top sizes of $1 \mathrm{~mm}$ and $3 \mathrm{~mm}$ for diffuse reflectivity (CIE $x$ value of about 0.3300 ). Right side (g-i): Differences of diffuse and specular reflectivity for a globe-top size of $3 \mathrm{~mm}$ and the phosphor concentration adjusted in order to achieve a CIE $x$ value of about 0.3800 for a diffuse reflectivity of $85 \%$. Top: reflectivity $85 \%$, middle: $70 \%$, bottom: $100 \%$.

Figure 7 compares the radiant flux ratios for the blue, the yellow and the total radiant fluxes of LED packages with a hemispherical globe-top in dependency of the globe-top size (all with respect to a globe-top radius of $1 \mathrm{~mm}$ ). As obvious, the larger the globetop radii the larger are the radiant fluxes. As evident from the right side image of Figure 7, the differences in the radiant fluxes of globe-tops with smaller and larger radii are the more pronounced the higher the phosphor concentration is, this means the lower the CCT values are. To conclude and contrary to colour maintenance, which is favoured by smaller globe-top sizes, in terms of the radiant fluxes a larger globe-top radius is advantageously.

In order to highlight the impact of the different surface reflectivities on the colour variations in detail, Figure 8 shows these variations with respect to MacAdam ellipses of step-size 2 (blue dotted), 4 (red solid) and 5 (gray dotted). The ellipses are centerd around a CIE $x$ value of about 0.3300 , which represents the reference configuration with a diffuse substrate surface reflectivity of $85 \%$. An increase or a reduction of the surface reflectivity to $100 \%$ and $70 \%$, respectively, causes colour variations, which match the outer limits of a MacAdam ellipse of step-size 2 for a globe-top radius of $1 \mathrm{~mm}$ and even of step-size 4 in case of a globe-top with a radius of $3 \mathrm{~mm}$ (for diffuse reflectivity, see left image of Figure 8). Using a surface with a specular instead of a diffuse reflectivity causes a shift of these deviations to the bluish (middle image of Figure 8). This means, the colour deviation is even more pronounced for a reduction of 


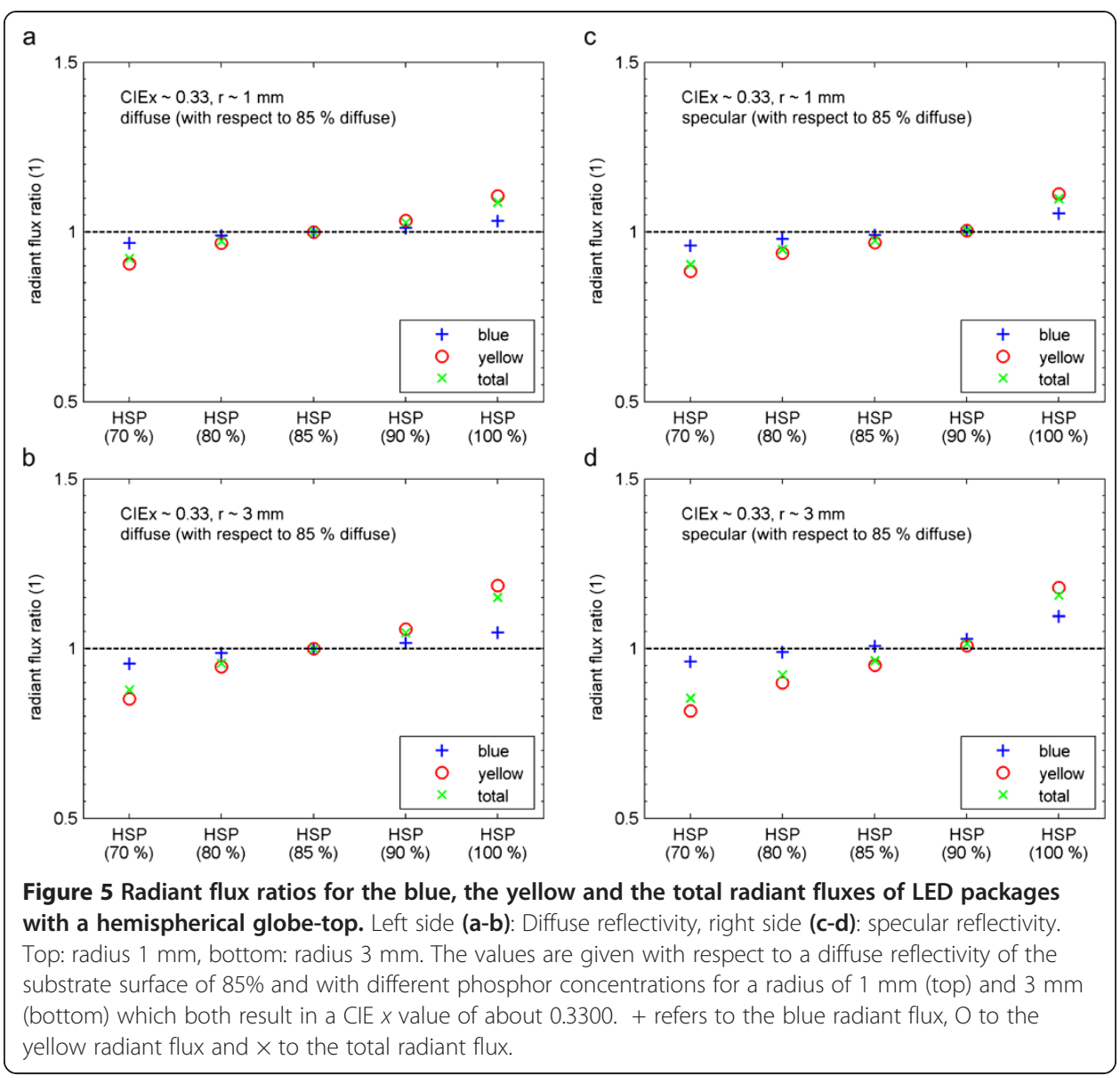

the surface reflectivity as compared to the case of a diffuse reflectivity of $85 \%$, but is diminished for an increase of the surface reflectivity. However, the relative colour deviations are also in case of a specular reflectivity quite similar to the relative deviations in case of a diffuse reflectivity. This is shown in the right image of Figure 8, for which the phosphor concentrations were varied so that also for a specular reflectivity a CIE $x$ value of about 0.3300 is achieved for a surface reflectivity of $85 \%$ (for $1 \mathrm{~mm} 5.17$ vol.\%, for $2 \mathrm{~mm} 2.29$ vol.\% and for $3 \mathrm{~mm} 1.49$ vol.\%). This behaviour is quite similar for globe-tops with phosphor concentrations giving reason for CIE $x$ values of about 0.3800 , despite the fact that the overall colour deviations are not that pronounced (see Figure 9).

Therefore, and as discussed above, from the viewpoint of CCT maintenance, a globetop with smaller size is preferable, since such a globe-top configuration shows the lowest deviation from the initial CIE $x$ value in case that the reflectivity of the substrate surface diminishes because of long term materials degradation.

However, this is contradictory to the request of higher radiant fluxes, which are favoured by larger globe-top sizes.

Anyhow, as mentioned above, besides the optical behaviour, also the thermal behaviour should be considered in a comprehensive discussion of optimized globe-top configurations. Due to the fact that the silicone matrix has a comparable low thermal conductivity (typically $0.2 \mathrm{~W} / \mathrm{mK}$ ) and due to the heat generated within the CCE upon colour conversion (Stokes shift, quantum efficiency lower than unity), there may be a notable thermal 


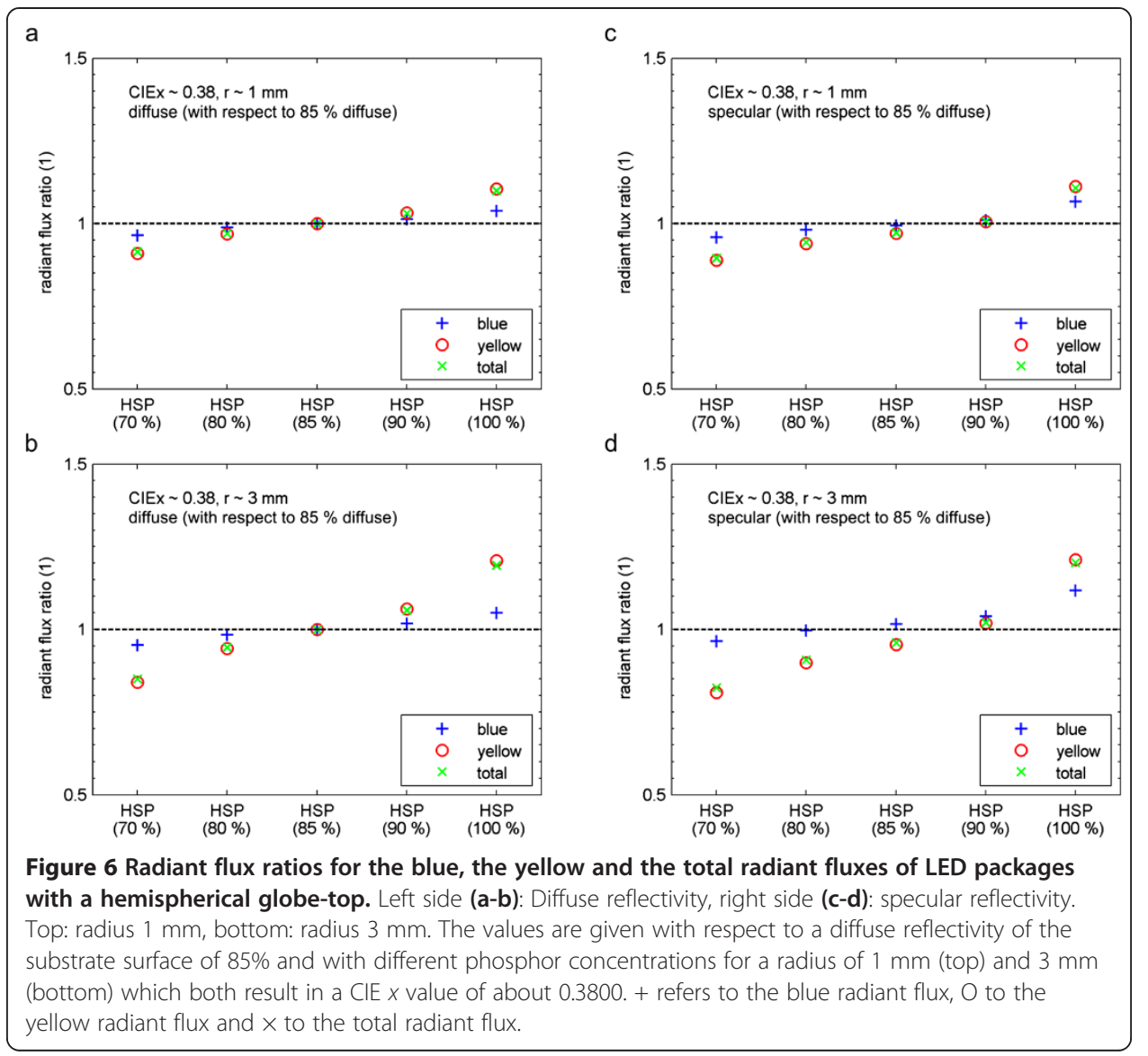

load of the CCEs upon device operation [25,30,36,37]. In order to study the impact of the different globe-top configurations on the respective thermal load, Figure 10 shows the calculated temperature profiles along the vertical directions of the LED packages. In this figure, a height $\mathrm{z}$ of $0 \mu \mathrm{m}$ in vertical direction coincides with the top of the PCB. As evident from the left image of Figure 10, which shows the temperature profiles for the three hemispherical globe-top configurations with radii of $1 \mathrm{~mm}, 2 \mathrm{~mm}$, and $3 \mathrm{~mm}$ for a CIE $x$ value of about 0.3300 under the assumption of a quantum efficiency of $100 \%$, the

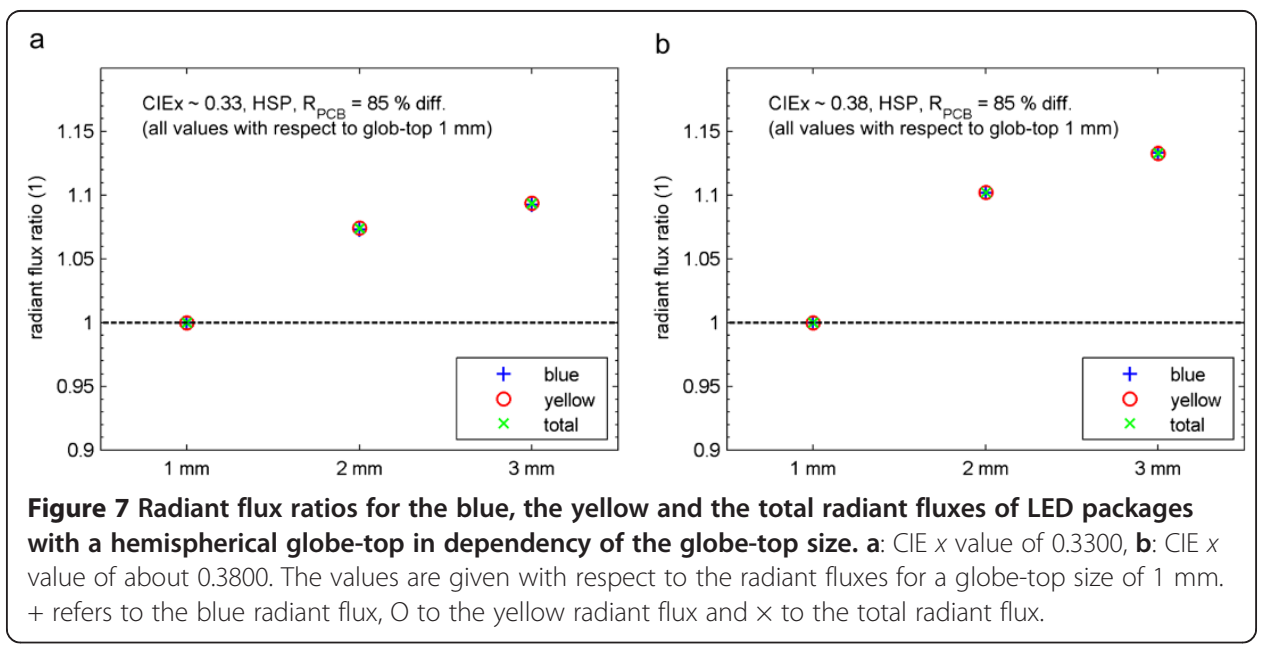




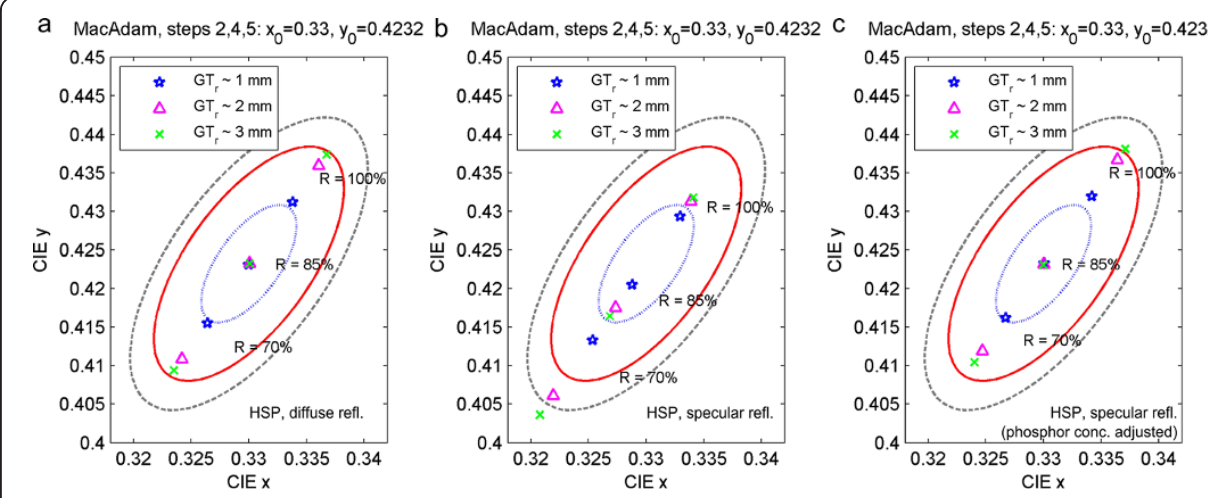

Figure 8 MacAdam ellipses of step-size 2, 4 and 5 centered around a CIE x value of about 0.3300 . $\mathrm{CIE} x$ values resulting for the different globe-top radii for diffuse reflectivity (left side, a), specular reflectivity (middle, b) and specular reflectivity under the assumption that the phosphor concentration is adjusted to result in a CIE $x$ value of about 0.3300 also for a specular reflectivity of $85 \%$ (right side, c). GT refers to the globe-top radius.

by far highest maximum temperatures are reached for the globe-top with a radius of $1 \mathrm{~mm}$. Such high maximum temperatures indeed may have some drawbacks upon device operation. For example, the luminescence intensity of most of the phosphor materials decreases with increasing temperature. Besides an overall reduction of the luminous efficacy, this may also cause some shifts of the chromaticity coordinates due to the lower fraction of yellowish emission. In addition, higher temperatures also enhance the risk for long term-materials degradation and deterioration of the optical properties. Therefore, from a thermal point of view, globe-tops with larger sizes are preferable in order to keep the thermal stress of the materials as low as possible. However, besides the overall temperature, also the variation of the temperature with increasing current is of importance. Generally, the higher the current the higher is the thermal load which is induced in the CCE. Considering the fact that, as mentioned above, for a Ce doped YAG phosphor a temperature variation of about $40 \mathrm{~K}$ may cause a colour deviation which matches the outer limits of a MacAdam ellipse of step 2 [25], larger temperature

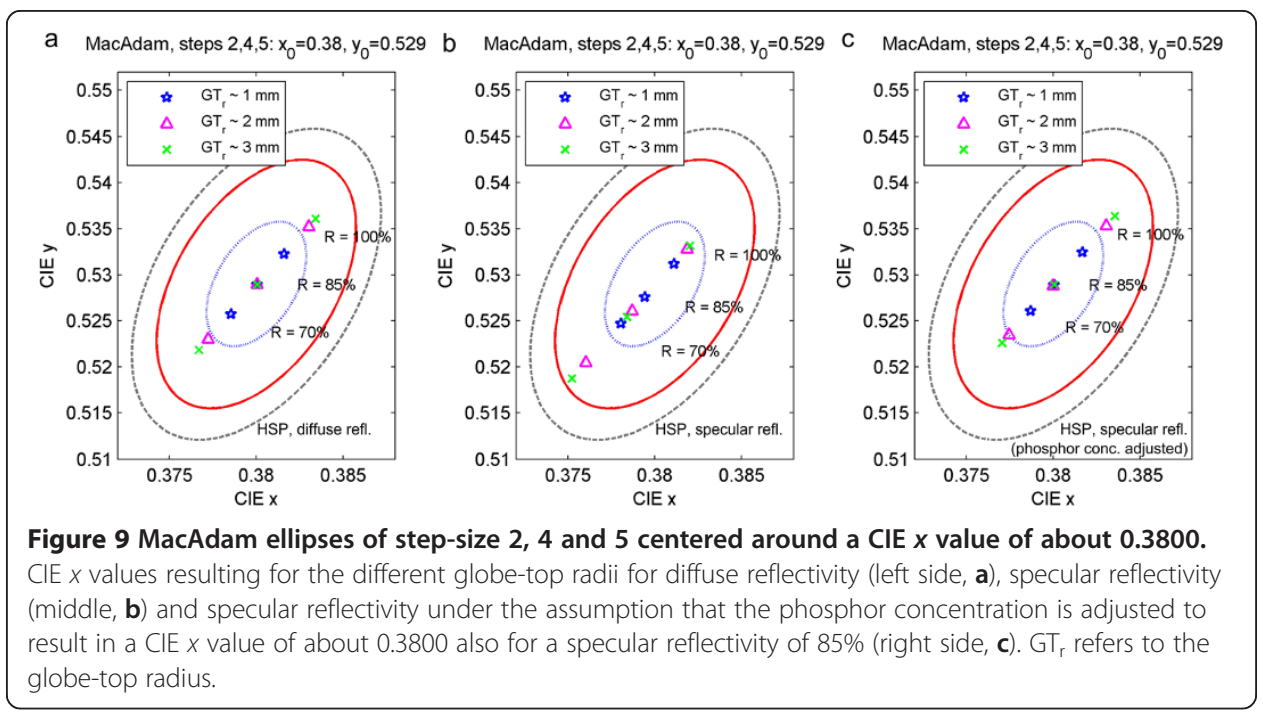



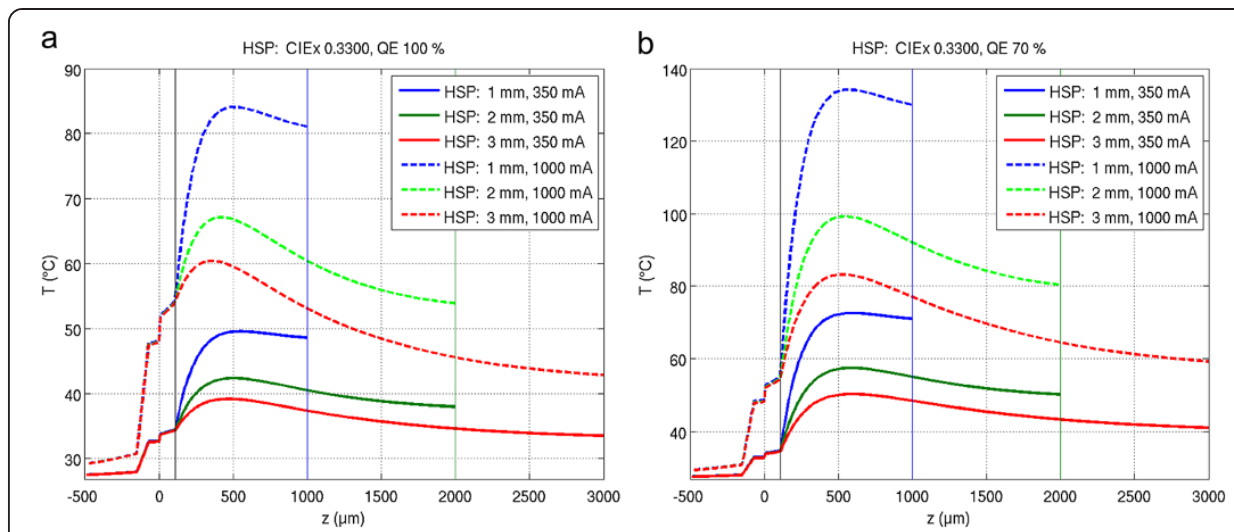

Figure 10 Line scans for the temperature profiles along the vertical directions of the LED packages (for the three radii and phosphor concentrations resulting in $\mathrm{CIE} \times$ values of about 0.3300 ) assuming that the assembly is mounted on a cooler with a constant temperature of $300 \mathrm{~K}$. The quantum efficiency of the phosphor is $100 \%$ (a) and $70 \%$ (b). The position $z=0 \mu \mathrm{m}$ coincides with the top surface of the PCB. HSP: $1 \mathrm{~mm}$ refers to a hemispherical globe-top with a radius of $1 \mathrm{~mm}$, similarly HSP: $2 \mathrm{~mm}$ and HSP: $3 \mathrm{~mm}$ to hemispherical globe-tops with radii of $2 \mathrm{~mm}$ and $3 \mathrm{~mm}$. Shown are the results for device operation at currents of either $350 \mathrm{~mA}$ or $1000 \mathrm{~mA}$.

variations may also cause notable colour variations. As obvious, going from $350 \mathrm{~mA}$ to $1000 \mathrm{~mA}$ causes a much larger temperature variation in case of the smallest globe-top radius in comparison with those of larger radii. This means that in particular globe-tops with small radii are prone to suffer from temperature dependent colour variations upon device operation at different currents.

These thermal impacts (higher maximum temperatures and larger temperature variations for different driving currents in case of smaller globe-top radii) again also dimish the aforementioned advantages of smaller globe-top radii in regard of CCT maintenance and restricts their applicability in particular to applications for which the current is not varied upon device operation.

\section{Conclusions}

As evident from these studies, which were performed for LED packages with CCEs in globe-top configuration, optimized CCE design in regard of CCT maintenance, radiant fluxes and low thermal load may be counteractive. Considering the fact that the reflectivity of the substrate surface is prone to some long term reduction because of materials degradation, a globe-top of smaller size seems to be preferable in regard of CCT maintenance. On the other hand, a smaller globe-top size gives reason for much higher maximum temperatures within the globe-top during device operation and much larger temperature variations for different driving currents, which again may induce pronounced colour variations and may amplify long-term materials degradation. A compromise based on globe-top configurations of medium sizes therefore seems to be the best strategy to meet all the requirements of colour maintenance, radiant fluxes, and low maximum temperatures at once.

Nonetheless, if only one of these parameters, like colour maintenance or high radiant fluxes is of particular importance for a specific application, the size of the globe-top can be adjusted to meet these requirements at best.

These results in particular highlight the complex interplay of the different effects that contribute to the colour conversion process and finally determine the luminous 
fluxes and the white light quality of phosphor converted LEDs and the need for the optimization of the CCEs in this regard.

Competing interests

The authors declare that they have no competing interests.

\section{Authors' contributions}

All authors participated in the conception of the manuscript. The optical and thermal simulations were mainly carried out by SS, CS and PF. The manuscript was drafted by FPW. All authors read and approved the final manuscript.

\section{Acknowledgements}

The authors gratefully acknowledge financial support from the "Neue Energien 2020" program, project number 827784, of the Austrian Climate and Energy Fund.

\section{Author details}

${ }^{1}$ Institute of Surface Technologies and Photonics, Joanneum Research, Forschungsgesm.b.H., Franz-Pichler Straße 30, A-8160 Weiz, Austria. ${ }^{2}$ Institute of Sensor and Actuator Systems, Vienna University of Technology, Gusshausstraße 27-29, A-1040 Vienna, Austria. ${ }^{3}$ Tridonic Jennersdorf GmbH, Technologiepark 10, A-8380 Jennersdorf, Austria. ${ }^{4}$ AT\&S Austria Technologie and Systemtechnik AG, Fabriksgasse 13, A-8700 Leoben, Austria.

Received: 8 April 2014 Accepted: 7 October 2014

Published online: 08 November 2014

\section{References}

1. Round HJ: A note on carborundum. Electrical World 1907, 49:309.

2. Zheludev N: The life and times of the LED - a 100-year history. Nature Photon 2007, 1:189.

3. Nakamura S, Senoh M, Mukai T: P-GaN/N-InGaN/N-GaN double eterostructure blue-light-emitting diodes. Jpn J Appl Phys 1993, 32:L8

4. Nakamura S, Mukai T, Senoh M: Candela-class high-brightness InGaN/AIGaN double-heterostructure blue-light-emitting diodes. Appl Phys Lett 1994, 64:1687.

5. Brodrick J: Solid-State Lighting: Technology at a turning point. http://apps1.eere.energy.gov/buildings/ publications/pdfs/ssl/feb2013_nema_brodrick.pdf

6. Narendran N, Deng L, Pysar RM, Gu Y, Yu H: Performance characteristics of high-power light-emitting diodes. Proc SPIE 2004, 5187:267.

7. Xiao H, Lu Y-J, Shih T-M, Zhu L-H, Lin S-Q, Pagni PJ, Chen Z: Improvements on Remote Diffuser-Phosphor-Packaged Light-Emitting Diode Systems. IEEE Photonics J 2014, 6:8200108.

8. Sommer C, Krenn JR, Hartmann P, Pachler P, Schweighart M, Tasch S, Wenzl FP: The effect of the phosphor particle sizes on the angular homogeneity of phosphor-converted high-power white LED light sources. IEEE $J$ Sel Top Quantum Electron 2009, 15:1181.

9. Liu Z-Y, Li C, Yu B-H, Wang Y-H, Niu H-B: Effects of YAG: Ce Phosphor Particle Size on Luminous Flux and Angular Color Uniformity of Phosphor-Converted White LEDs. IEEE/OSA J Disp Technol 2012, 8:329.

10. Zheng H, Luo XB, Hu R, Cao B, Fu X, Wang Y, Liu S: Conformal phosphor coating using capillary microchannel for controlling color deviation of phosphor-converted white light-emitting diodes. Opt Express 2012, $20: 5092$.

11. You JP, Tran NT, Shi FG: Light extraction enhanced white light-emitting diodes with multi-layered phosphor configuration. Opt Express 2010, 18:5055.

12. Hu R, Luo XB, Liu S: Study on the optical properties of conformal coating light-emitting diode by Monte Carlo simulation. IEEE Photonics Technol Lett 2011, 23:1673.

13. Yang T-H, Chen C-C, Chen C-Y, Chang Y-Y, Sun C-C: Essential Factor for Determining Optical Output of Phosphor-Converted LEDs. IEEE Photonics J 2014, 6:8200209.

14. Sun C-C, Chen C-Y, Chen C-C, Chiu C-Y, Peng Y-N, Wang Y-H, Yang T-H, Chung T-Y, Chung C-Y: High uniformity in angular correlated-color-temperature distribution of white LEDs from 2800K to 6500K. Opt Express 2012, 20:6622.

15. Sommer C, Hartmann P, Pachler P, Schweighart M, Tasch S, Leising G, Wenzl FP: A detailed study on the requirements for angular homogeneity of phosphor converted high power white LED light sources. Opt Mater 2009, $31: 837$.

16. Hu R, Luo XB, Feng H, Liu S: Effect of phosphor settling on the optical performance of phosphor-converted white light-emitting diode. J Lumin 2012, 132:1252.

17. Chang-Chien CL, Huang YC, Yip MC, Fang W: 'Flip glass substrate' package technology for LED yield and performance enhancement. J Micromech Microeng 2012, 22:105039.

18. Sommer C, Reil F, Krenn JR, Hartmann P, Pachler P, Hoschopf H, Wenzl FP: The Impact of Light Scattering on the Radiant Flux of Phosphor-Converted High Power White Light-Emitting Diodes. J Lightwave Technol 2011, 29:2285.

19. Liu Z-Y, Li C, Yu B-H, Wang Y-H, Niu H-B: Uniform white emission of WLEDs realized by multilayer phosphor with pyramidal shape and inversed concentration distribution. IEEE Photonics Technol Lett 2012, 24:1558.

20. Lin M-T, Ying S-P, Lin M-Y, Tai K-Y, Tai S-C, Liu C-H, Chen J-C, Sun C-C: Ring Remote Phosphor Structure for Phosphor-Converted White LEDs. IEEE Photonics Technol Lett 2010, 22:574.

21. Sommer C, Reil F, Krenn JR, Hartmann P, Pachler P, Tasch S, Wenzl FP: The impact of inhomogeneities in the phosphor distribution on the device performance of phosphor-converted high power white LED light sources. J Lightwave Technol 2010, 28:3226.

22. Hsiao S-L, Hu N-C, Cornelissen H: Phosphor-converted LED modeling using near-field chromatic luminance data. Opt Express 2013, 21:A250. 
23. Li Z-T, Tang Y, Liu Z-Y, Tan Y-E, Zhu B-M: Detailed Study on Pulse-Sprayed Conformal Phosphor Configurations for LEDs. IEEE/OSA J Disp Technol 2013, 9:433.

24. Chen C-Y, Yang T-H, Hsu C-H, Sun C-C: High-Efficiency White LED Packaging With Reduced Phosphor Concentration. IEEE Photonics Technol Lett 2013, 25:694.

25. Fulmek P, Sommer C, Hartmann P, Pachler P, Hoschopf H, Langer G, Nicolics J, Wenzl FP: On the Thermal Load of the Color-Conversion Elements in Phosphor-Based White Light-Emitting Diodes. Adv Opt Mater 2013, 1:753.

26. Sommer $C$, Hartmann P, Pachler $P$, Hoschopf H, Wenzl FP: White light quality of phosphorconverted light-emitting diodes: A phosphor materials perspective of view. J Alloys Compd 2012, 520:146.

27. Sommer C, Fulmek P, Nicolics J, Schweitzer S, Nemitz W, Hartmann P, Pachler P, Hoschopf H, Schrank F, Langer G, Wenzl FP: Thermal and optical aspects of glob-top design for phosphor converted white LED light sources. Proc SPIE 2013, 8835:88350J.

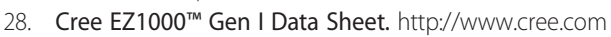

29. Kim H, Kim KK, Lee SN, Baik KH: Design and fabrication of vertical-injection GaN-based light-emitting diodes. Opt Express 2011, 19:A937.

30. Wenzl FP, Sommer C, Hartmann P, Pachler P, Hoschopf H, Langer G, Fulmek P, Nicolics J: The impact of the non-linearity of the radiant flux on the thermal load of the color conversion elements in phosphor converted LEDs under different current driving schemes. Opt Express 2013, 21:A439.

31. Open source software packages: Gmsh: a three-dimensional finite element mesh generator with built-in preand post-processing facilities, GetDP: a general environment for the treatment of discrete problems. http://www.geuz.org

32. Geuzaine C, Remacle J-F: A 3-D finite element mesh generator with built-in pre- and post-processing facilities. Int I Numer Methods Eng 2009, 79:1309.

33. Enigl R: Master Thesis, AEM-ISAS. University of Technology Vienna; 2000

34. Physical properties of CE:YAG. http://www.scientificmaterials.com/products/ce-yag.php

35. Material safety data sheet Polydimethylsiloxane. http://www.essentialingredients.com/msds/Element $\% 2014 \%$ 20PDMS\%20350.pdf

36. Yan B, Tran NT, You J-P, Shi F-G: Can Junction Temperature Alone Characterize Thermal Performance of White LED Emitters? IEEE Photonics Technol Lett 2011, 23:555.

37. Hu R, Luo XB, Zheng H: Hotspot location shift in the high power phosphor converted white light-emitting diode package. Jpn J Appl Phys 2012, 51:09MK05.

doi:10.1186/s40539-014-0018-1

Cite this article as: Schweitzer et al: A comprehensive discussion on colour conversion element design of phosphor converted LEDs. Journal of Solid State Lighting 2014 1:18.

Submit your manuscript to a SpringerOpen ${ }^{\circ}$ journal and benefit from:

- Convenient online submission

- Rigorous peer review

- Immediate publication on acceptance

- Open access: articles freely available online

- High visibility within the field

Retaining the copyright to your article 